\title{
NHD. BRAUT IN DEN GERMANISCHEN SPRACHEN.
}

Oben s. 6 ff. hat sich ergeben, dass in der alts. und ags. dichtung brî̀, brýcl 'gattin, eheweib' bedeutet. Ohne jede beschränkung auf das lebensalter beżeichnet das wort die frau, insofern sie mit dem manne in der ehe, in geschlechtlichem umgange verbunden ist. Für uns handelt es sich nun um die frage, wie sich dazü der gebrauch in den übrigen germanischen sprachen stellt und was wol die grundbedeutung und etymologie des wortes sein mag. Zur beantwortung derselben liegt für die einzelnen dialekte reiches material in den wörterbüchern bereit; hinsichtlich der etymologie verweise ich auf die arbeit von Wiedemann, Bezzenb. Beitr. 27 (1902), 205 ff., wo die frühere etymologische literatur verzeichnet und erörtert ist.

Als die verbreitetste bedeutung von nhd. braut in den germanischen sprachen ist zunächst festzustellen: 'neuvermählte, junge frau', und zwar vom tage der hochzeit an, diesen inbegriffen. Ausschliesslich diese bedeutung hat noch jetzt das neuengl. bride, vgl. z. b. Grieb-Schröer, Wb. 1, 138: 'bride die braut (und zwar nicht wie im deutschen $=$ die verlobte, sondern nur die braut am hochzeitstage, unmittelbar vor, während oder nach der hochzeit, ferner abweichend vom deutschen auch noch:) die junge kürzlich verheiratete frau'1). $\mathrm{Zu}$ dieser be-

1) Vgl. Murray, Dict. 1, 1095, wo auch die geschichte erzählt wird, dass Gladstone im parlament entsetzen erregt habe, als er auf eine verlobte prinzessin das wort bride, also in deutscher weise, anwandte. Er musste das dann in bride elect verbessern. Im deutsch-engl. encycl. wörterbuch von Muret-Sanders s. 387 steht als bedeutung von nhd. braut: 1) verlobte: betrothed (or affianced, intended, futuve) bride, intended wife, givl engaged; seine braut: his intended; 2) am hochzeitstage und noch kurz darauf bride (entsprechend bräutigam Muret-Sanders s. 388). Wo also englisch bride für 
deutung gehört noch ne. (aber veraltet) bridalry 'flitterwochen' und to bricle 'heiraten'. Dass diese ne. bedeutung auch mit dem altdeutschen gebrauche sich teilweise deckt, ist bekannt und eben dadurch ist ja Behaghel (bez. Roediger) zu seinem oben besprochenen misverständnis des alts. brûd gekommen.

Nachdrücklichst auszuschalten ist aber vor allem die bedeutung der nhd. schriftsprache, wonach braut die verlobte ist, d.h. das versprochene mädchen, von der formellen verlobung an bis zum hochzeitstage, aber auch noch diesen selbst eingeschlossen bis zu dem rechtlich bindenden act vor standesamt und kirche. Von da ab wird aus der braut die 'junge frau' nach unserem heutigen sprachgebrauch, der damit also dem älteren diametral entgegengesetzt ist. Berührungspunkt beider ist der tag der hochzeit. Diese besonderheit der nhd. bedeutung des wortes braut ist deshalb so scharf hervorzuheben, weil in den darstellungen und wörterbüchern meist die verwirrung herscht, dass dem altgerm. worte daneben auch die bedeutung 'verlobte' zugeschrieben wird.') So z.b. Heyne, DWb. 1, 483: braut 'verlobte ..., ahd. prût, mhd. brût auch junge frau, neuvermählte'; Lexer, Mhd. wb. 1, 373 brît: 'die verlobte oder kürzlich vermählte braut, junge frau'; und Gundermann, Zs. f. deutsche wortf. 1, 245, wo er die verschiedenen bedeutungen sondert; stellt als erste voran: 'verlobte, nhd. in der schriftsprache ausschliesslich, mhạ. br $\hat{u} t$, ahd. brût, ags. brýd, an. brúðr; im got. brupfaps brautherr, bräutigam'. Hier und anderwärts wird also dem ags. brýd, altn. brúdr etc. neben der anderen auch die bedeutung 'verlobte'. beigelegt. Das ist aber ganz falsch. Die braut als verlobte ist im altn. nur festarkona, festarmoer, der nhd. bräutigam ist altn. festarmadr und so bis in die neunord. sprachen, z. b. schwed. fästmö,

unsern speciell nhd. begriff (= verlobte) gebraucht werden soll, muss es durch ein attribut als diejenige bestimmt werden, welche in zukunft einmal bride werden soll.

1) Diesen fehler vermeidet aber Paul, Dtsch. wb: s. v. braut: 'grundbedeutung "neuvermählte", sie wurde so bezeichnet am hochzeitstage wie noch jetzt, auch bevor die vermählung schon vollzogen war; von daher ist es dann in die bedeutung "verlobte" übergegangen. Die beziehung auf die vollzogene vermählung ist noch deutlich in zuss. wie brautkammer, brautbett, brautnacht.' 
dän. foesteme, kjoereste, forlovede: erst mit dem hochzeitstage tritt dän. und schwed. brud in seine rechte. Das ags. scheint für verlobte ein eigenes substantivum nicht gehabt zu haben, für verloben wird weddian, beweddian gebraucht, verlobung ist weddung, beweddung; die ne. entsprechung ist to betroth und betrothal (vgl. Murray 1,832), die braut muss ne. entweder durch das französische fremdwort fiancée, oder die oben s. 30, anm. besprochenen umschreibungen ausgedrückt werden.

Auf deutschem gebiete war in alter zeit mahlian das verbum des. versprechens, verlobens. So heisst es alts. Hel. 253 ff.: Sea en thegain habda Joseph gimahlit ... thea Dauides dohter: that unas so diurlic unif, idis antheti. Also alts. anthêt $i$ îf ist die braut im nhd. sinne. Und Hel. 297 heisst es, dass Joseph thea magad habda, thea idis anthetea giboht im te brudiu; also er hatte vor, seine braut zum weibe zu machen. ${ }^{1}$ ) Und im ahd. und mhd. ist ebenfalls mahalen, mehelen das wort des verlobens. ${ }^{2}$ ) Belege bei Graff 2,651 f. So z.b. Wiener Genesis (Fdgr. 2, 14, 14) damite (mit dem ringe) der man spulget sîn wî̀b mahileri; O. 1,8,1 ther man ther thaz wî̀ mahalta (Joseph). Maria hätte nach ahd. und mhd. sprachgebrauch niemals als Josephes brût bezeichnet werden können ${ }^{3}$ ), wol

1) Nur an diesen beiden stellen ist anthêti in den hss. überliefert. An zwei weiteren stellen, wo Heyne anthêti in bezug auf verheiratete frauen in seinen text setzt: Hel. 506 und 2707, ist dieses gar nicht belegt, sondern beide male an ehti $\mathrm{C}$ 'im besitz', währeud $\mathrm{M}$, dem diese wendung unbekannt gewesen zu sein scheint, anthehti, bez. antehti schreibt; Behaghel liest beide male richtig nach $\mathrm{C}$ an êthti.

2) Im mhd. wird auch vestenen, bevestenen im sinne von 'verloben' gebraucht, s. Mhd. wb. 3, 277; die belege gehören der früheren zeit an: spätmhd. kommt, während gleichzeitig vermählen seine bedeutung verschob, verloben auf, über dessen entwicklung $\mathrm{s}$. DWb. 12, $816 \mathrm{ff}$. Wie es scheint, ist der ausdruck mehr md., bei Luther schon ganz häufig; doch hat dieser im gleichen sinne auch vertrauen, vgl. Luc. 2, 5 mit Maria seinem vertrauten

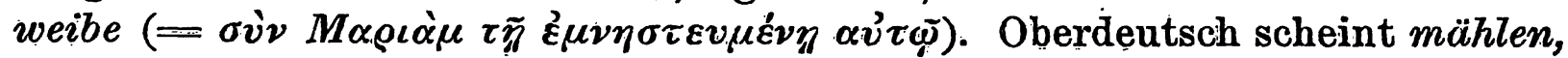
vernıählen länger bestand gehabt zu haben, vgl. statt Luthers vertrauen bei Ẹck vermählen und Züricher Bibel vermächlen (Kluge, Luther-Lessing s. 82). S. auch DWb. 6,1455 s. v. mählen. Daselbst z. b. aus. der Bibel 1483 der do hat gemehelt ein weib (qui despondit uxorem 5. Mos. 20, $7=$ Luther: welcher ein weib im vertrauet hat).

3) Dagegen 0. 2, 8 in der erzählung der hochzeit von Kana erscheint natürlich brût und brûtigono. 
aber als Josephes gimahaltiu, vgl. T. 5, 12 mit Mariun imo gimahaltero gimahhun. Das eigentliche ahd. substantivum für nhd. 'bräutigam' und 'braut' ist ahd. gimahalo und gimahala. So ist in einer Reichenauer glosse zu Matth. 1, 16 Joseph virum Mariae das wort virum Gl. 1, 708, 27 mit gimahelon glossiert, weil er nach der correcten theologischen auffassung nur ihr verlobter war. Ebenso ist in demselben glossar (dem hier noch andere hss. zur seite stehen) die stelle Matth. 1,20 Mariam conjugem tuam das conjugem durch dogmatisch correcteres gemahalun widergegeben, Gl. 1, 709, 6.1) So ist denn auch noch mhd. der gemahele und diu gemahele der richtige ausdruck für nhd. bräutigam und braut, wie die wörterbücher ausweisen. Besonders lehrreich ist die anwendung im armen Heinrich, wo Heinrich das mädchen in liebkosendem scherz sîn gemahele nennt (a. H. 341 u. ö.). Zarncke im Mhd. wb. 2,20 übersetzt das sehr richtig mit 'mein bräutchen' und trûtgemahel mit 'liebe braut'. Aber schon im mhd. wird daneben gemahel und gemahele auch für verheiratete gebraucht, welche bedeutung ja bekanntlich im nhd. seit dem 15.16. jh. die ursprüngliche völlig verdrängt hat.2) Der bedeutungsübergang war

1) Solche stellen dürfen also nicht gebraucht werden, um für ahd. gemahala die bedeutung conjux zu erweisen. Ueberhaupt muss ein für alle mal gewarnt werden, ahd. glossen zu bestimmten schriftstellern bedeutungsgeschichtlich zu verwerten, ohne die stelle im zusammenhange des textes zu prüfen. - Zur vergleichung möge darauf hingewiesen werden, dass T. in obigen stellen wörtlich übersetzt, also Mt. 1, 16 virum Mariae mit gomman Mariun (ebenso v. 19) und 20 conjugem mit Mariun thina gimahtiun widergibt, während der glossator nachgedacht hat.

2) Vielleicht sind schon einige spätahd. stellen für diesen bedeutungswandel in anspruch zu nchmen. Bei N. Marc. Cap. (ed. Piper 1,694, 18) ișt einmal conjuga mit sînero gemâlun umschrieben. Und Williram im Hohen liede übersetzt das sponsa seiner quelle regelmässig mit gemáhela (z. b. Cantic. 4, 8-12), während Luther dem alten und dem allgemein kirchlichen sprachgebrauche gemässer braut dafür anwendet. - Interessant ist auch der beleg aus den Windberger psalmen, wo ps. 18, 6 et ipse tanquam sponsus procedens de thalamo suo iubersetzt wird mit unde er selbe also der gemahele furegeente von bette sinem, während $N$. richtiger hat unde er selbo gieng uz also briutegomo uzer sinero briutechamero. Aber diese vereinzelten fälle lassen auch andere deutungen zu, vgl. unten s. 35. Reiches material zur bedeutungsgeschichte von gemahl und der neubildung gomahlin s. in den artikeln $\mathrm{R}$. Hildebrands, DWb. 4, 1, 2, 3150 ff.

Beiträge zur geschichte der deutschen sprache. XXXII. 
vielleicht auch sachlich dadurch bedingt, dass auf das feierliche zusammensprechen in vielen fällen der vollzug der ehe unmittelbar folgte, also ein brautstand in unserem sinne entfiel.

Dass man entgegen diesen feststellungen so allgemein in den wörterbüchern dem altdeutschen und ags. worte brût auch die bedeutung von nhd. braut $=$ 'verlobte' zuschreibt, dazu ist der anlass der, dass sowol in ags. als in ahd. glossen ags. brýd, ahd. brût bez. brýdzuma, brûtigomo als die regelmässigen entsprechungen von lat. sponsa, sponsus auftreten. 1) Und ebenso wird in der biblischen übersetzungsliteratur ags. wie ahd. das sponsa, sponsus der vulgata widergegeben. Ja auch O. 2, 13, 9 gibt Joh. 3, 29 qui habet sponsam sponsus est durch Ther brut habet, in unar min, ther scal ther brútigomo sin.

Hier rächt sich nun der umstand, dass wir zu sehr im. sprachgefühl des clässischen lateins befangen sind und die spätlateinische entwicklung $\mathrm{zu}$ wenig beachten. Gewis heisst im classischen latein sponsa, sponsus der bedeutung des verbums spondeo entsprechend das, was wir nhd. durch braut und bräutigam widergeben. ${ }^{2}$ ) Aber im nachclassischen, christlichen latein hat sich dieselbe bedeutungsverschiebung vollzogen, die wir soeben bei ahd. gimahalo, gimahala beobachtet haben, welche ursprünglich dem classisch-lat. sponsa, sponsus entsprachen, heute äber zur ausschliesslichen. bedeutung von maritus und uxor gelangt sind. Genau so weit ist das latein nur im französischen gegangen, wo époux und épouse völlig dem nhd. gemahl und gemahlin gleichstehen. Aber im mittellatein ist sponsus und sponsa doch schon auf dem standpunkte angelangt, den heute noch das italienische zeigt, wo zwar plur. sposi schlechthin 'ehegatten' bedeutet, aber doch die singulare sposa und sposo auch noch die bedeutung des altdeutschen brût

1) Für das ags. vgl. in Wright-Wülker, Anglosaxon and old english vocabularies (1883) $1,171,7.9 ; 277,19.20 ; 310,12.13$. Doch steht daneben auch bryd für lat. nimpha 171, 17, nupte 456,25 , brydzuma für procus 42,5 . 466, 32, 528, 4. - Für das ahd. vgl. Graff 3, 293 unter brût mit den übersetzungen nupta, sponsa und brûtigomo 4,200 f. mit sponsus, auch dem in lat. dichtern dafür gebrauchten procus.

2) Forcellini, Lex. erklärt: sponsa est mulier alicui promissa in matrimonium, pacta, sperata et nondum uxor; - sponsus est is qui desponsus est, nondum maritus, Cic. Horat. 
und brûtigomo festhalten, d. h. die neuvermählten, jungen eheleute, mit einschluss des hochzeitsfestes, bezeichnen. Dagegen den begriff des classisch-lat. sponsa und des nhd. brant drückt das italienische durch promessa sposa oder fidanzata aus, entsprechend das masc. promesso sposo oder fidanzato und der plural promessi sposi $=$ nhd. 'brautleute, verlobte'.

Die bedeutungswandlung von sponsus, sponsa scheint schon in den ersten jahrhunderten nach Christus vollzogen zu sein. Forcellini sagt, nachdem er die classische bedeutung angegeben hat (s. vor. anm.): 'sponsa dicitur etiam de uxore, sicut sponsus de marito'. Er verweist dabei auf die vulgata (Matth. 25, 1) und auf inschriften (z. b. Julia Fortunata vixit annis XXVIII cum sponso suo), woselbst also schon die volle bedeutung 'maritus' erreicht ist. Doch ist als die eigentliche geltung von sponsa, sponsus in der vulgata die des ahd. mhd. brût, brûtigomo zu bezeichnen, d.h. die neuvermählten mit einschluss der hochzeitsfeier. Wenn also z. b. in den Monseeer fragm. XX (Hench) Matth. 25, 1 exierunt obviam sponso et sponsae übersetzt wird mit ficorun uz ingegin brutigomin enti bruti, so entspricht diese übersetzung genau dem sinne des lateinischen: wir haben deshalb keine berechtigung, ahd. brût dem nhd. braut gleichzustellen. Und wenn Williram, wie oben (s. 33, anm.2) erwähnt, das sponsa in Cantic. mit gemáhela widergibt, so kann ihm, dem classisch gebildeten, schon dasselbe passiert sein, wie unseren heutigen lexicographen: er hat sich vielleicht durch die ihm aus dem classischen latein bekannte grundbedeutung von sponsa verleiten lassen ${ }^{1}$ ), nicht das der bedeutung der vulgata eigentlich entsprechende brût zu setzen. Denn die auf dem Hohen lied und der geschichte von den klugen und törichten jungfrauen (Matth. 25) beruhende kirchliche anschauungsweise vom.himmlischen bräutigam und der kirche als seiner braut, resp. der einzelnen seele, der nonne, als braut Christi ist doch viel sinnlicher gemeint, als die anwendung des nhd. braut und bräutigam es uns heute fühlen lässt: es sind vielmehr die geistlichen jungen eliegatten, der sponsus und

1) Auch das in der vulgata übliche despondere und desponsare für 'verloben' konnte bei sponsus, sponsa von neuem die alte bedeutung wider wachrufen. 
die sponsa der vulgata, so wie auch noch in der französischen kirchlichen terminologie hier époux und épouse für Christus und die kirche, oder die nonne, angewendet wird (vgl. Littrẹ, Dict. 1, 2, 1470). ') . Dem entspricht in dér mhd. geistlichen terminologie brût und briutgome. Das gegenstück ist die bekannte mhd. schelte des tiuvels brût, wodurch eine wéibsperson als teufelsbuhle gekennzeichnet wird, so wie auch in Nib. 417, 4 . 426, 4 (L.) des tiuvels wîp und des ïbelen tiuvels brît als gleichbedeutend von Brünhild gesagt werden.

Müssen wir sonach den begriff 'verlobte, versprochene' von dem worte $b r \hat{u} t$ in allen älteren germanischen sprachen vollständig fernhalten, als dessen wesentliches moment vielmehr der vollzug der ehe zu gelten hat, so ergibt sich daraus von selbst, dass die etymologische verknüpfüng mit scr. brávī-ti 'er spricht' abzuweisen ist. Nach dieser von Torp, Uhlenbeck und Hirt gleichzeitig aufgestellten etymologie sollte brüđiverbalabstractum 'das sprechen' sein, woraus sich die bedeutung 'verabredung, versprechung, verlobung' entwickelt hätte, und daraus wäre dann die germ. concrete anwendung erflossen. Trotz der einwendungen von Wiedemann a. a. 0. s. 206, der hervorhebt, dass scr. bráviti und composita nur 'sprechen', nirgends aber 'versprechen, verloben' bedeuten, hält Uhlenbeck, Beitr. 30, 271 seine etymologie aufrecht. Sie ist aber nach der bedeutung unmöglich, wie jede andere etwa aufzustellende etymologie, die von dem specifisch nhd. begriffe 'verlobte' ausgienge.

Nachdem also die besondere nhd. bedeutung ausgeschlossen ist, suchen wir nun den altgermanischen inhalt des wortes schärfer zu fassen. Wir fangen mit dem gotischen an, dessen als simplex nur einmal (Matth. 10,35) belegtes brûps fälschlich allgemein als 'schwiegertochter' aufgefasst wird. Nein, got,

1) Damit identisch sind die englischen religiösen termini the heavenly bridegroom - für Christus und bride (or spouse) of Jesus Christ für kirche oder nonne (Muret-Sanders a.a.o.), welche sonach für das ne. sprachgefühl etwas ganz anderes besagen, als unsere entsprechenden nhd. ausdrücke heute für uns. Doch tritt die alte bedeutung noch klar hervor, wenn es bei Schiller im Ritter Toggenburg heisst: 'Die ihr suchet trägt den schleier, ist des himmels braut, gestern war des tages feier, der sie gott getraut'. Die einsegnung der nonne gilt als geistliche vermählung. 
brûps bedeutet genau dasselbe wie ahd. brût. Ganz abgesehen davon, dass das compos. brûpfaps Mt. 9, 15; Mc. 2, 19; Luc. 5,

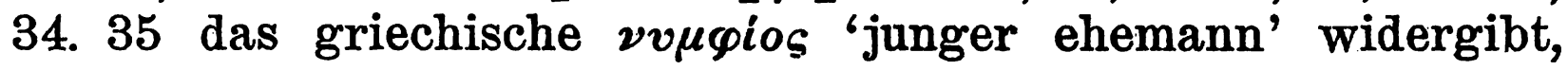
also dem ahd. brûtigomo entspricht, so ist auch jenes got. brûps die übersetzung des griech. v'v́ $\mu \eta \eta$. Und dieses ist die genaue entsprechung des ahd. mhd. brût, es bedeutet 'die neuvermählte, junge frau' die zeit des hochzeitsfestes mit ein-

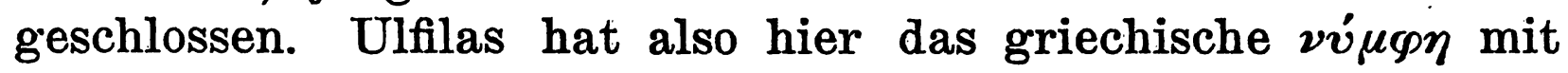
dem diesem worte in seiner regulären bedeutung entsprechenden gotischen brûps übersetzt. Nun hat allerdings griech. vísun Mt. 10, 35 (und an der parallelstelle Luc. 12,53) nach dem zusammenhange die occasionelle bedeutung 'schwiegertochter' '), eine bedeutung, welche von dem griechischen víurs in den wörterbüchern nur aus diesen neutestamentlichen stellen belegt wird. 2) Das altgriechische hatte für schwiegertochter in vvós die regelrechte entsprechung des aind. snušá. Wenn in der griech. bibel $\nu v^{\prime} \mu g: \eta$ statt dessen angewandt wird, so ist diese übertragung ja sachlich naheliegend, da die junge frau in das

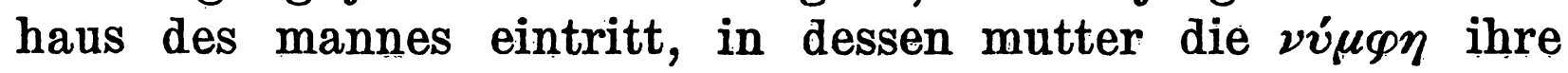
schwiegermutter findet. ${ }^{3}$ ) So wäre es an sich nicht aus-

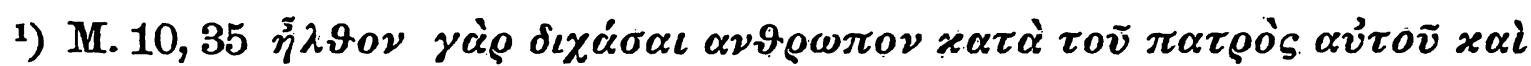

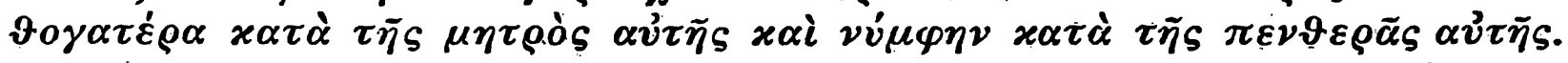

2) Vgl. z. b. Stephani thesaurus gr. linguae 5, 1601 ff.; Pape, Griech.-d. handwörterb. $2,263$.

3) Im neugriechischen ist diese übertragung sogar fest geworden. Vgl. z. b. Byzantius, Dictionn. Grec-français (Ath. 1856) s. 297: 'v́́ $\mu \varphi \eta$ 1) nymphe (mythol.) 2) la (nouvelle) mariée, l'épousée 3) bru, belle fille, belle soeur.' Aber im älteren griechisch ist die bedeutung ausserhalb der bibel bisher nicht nachgewiesen. Und es scheint, dass im bibelgriechischen die übertragung von $\nu v ́ \mu \varphi \eta$ auf die bedeutung 'schwiegertochter' erst durch das hebräische veranlasst ist. Die beiden evangelienstellen sind nach-

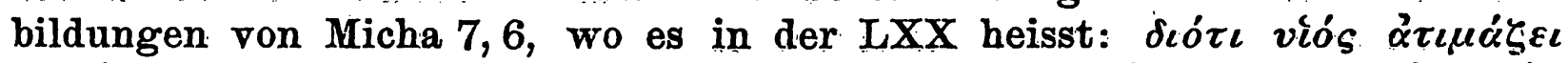

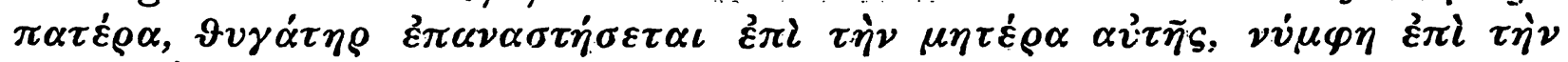
$\pi \varepsilon \nu \vartheta \varepsilon \rho \dot{\alpha} \nu \alpha \dot{v} \tau \tilde{\eta} \varsigma$. Das hebräische wort, welches an dieser stelle dureh $\nu \dot{v} \mu \varphi \eta$ gegeben wird, ist (kallāh), ebenso an andern stellen des A.T., wo Luther 'schnur' übersetzt (z. b. Gen. 38, 11. 24). Die grundbedeutung dieses hebräischen wortes ist aber $\nu v^{\prime} \mu \varphi \eta$ im siune des ahd. brût $=$ vulgata sponsa: in den betreffenden stellen des Hohen liedes steht stets hebr. welches in der LXX ebenfalls durch $\nu \dot{v} \mu \varphi \eta$, wie in der vulgata durch sponsa gegeben wird. Es haben also die LXX-übersetzer statt des in der griechischen prosa fehlenden einfachen wortes für den begriff 'schwiegertochter' (s. folg. anm.) in nachahmung des hebräischen doppelsinnes von 
geschlossen, dass auch im gotischen freien sprachgebrauch statt der wahrscheinlich noch vorhandenen entsprechung des ahd. snur, ags. snoru - gelegentlich einmal brûps hätte gebraucht werden können, wo es sich wie in Matth. 10,35 um die in das haus der eltern eingetretene 'junge frau' des sohnes handelte. Aber bewiesen wird das durch unsere stelle keinesfalls, da diese der eigenartigen anwendung des griech. $\nu \dot{v} \mu \varphi \eta$ verdankt wird. Hätte im N.T. vvós gestanden ${ }^{1}$ ), so würde uns wol auch das echt gotische wort *snuzô für schwiegertochter erhalten sein. ${ }^{2}$ ) Wir werden also vorsichtiger handeln, wenn wir auch in unseren gotischen wörterbüchern brûpss mit 'junge frau' übersetzen und dabei auf die eigentümliche anwendung von $v \tilde{v} \mu \varphi \eta$ im griech. original und dessen got. widergabe durch brîps verweisen.

Mit dem gotischen in beziehung stehen sicher auch die belege unseres wortes aus der römischen soldatensprache, deren älteste aus dem 3. und anfang des 4.jh.'s A. v. Domaszewski auf drei lateinischen soldateninschriften aus Bulgarien, Serbien und Kärnten nachgewiesen hat. Diese belege sind also um ein jahrhundert älter als Ulfilas. Die älteste lat. form des wortes ist brutis (bez. brutes), später bruta. Aber auch in das

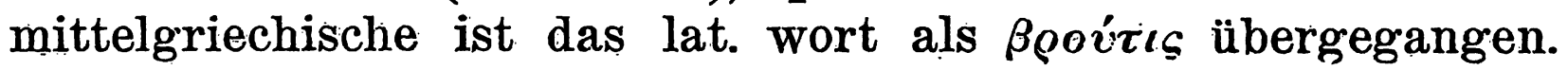
Ueber diesen gegenstand handelt zusammenfassend G. Gundermann, 'Das deutsche wort braut bei Römern und Griechen', Zs. f. deutsche wortforsch. 1,240 ff. und speciell über mgriech.

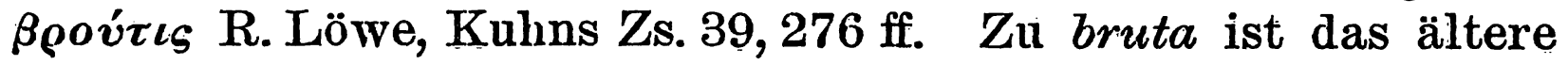
lat. brutis gemäss Gundermanns treffender erklärung nach analogie der fem. auf a umgebildet worden, wie nepta aus älterem neptis und nura aus nurus.

Zunächst ist die form des lat. brutis zu erörtern. Die

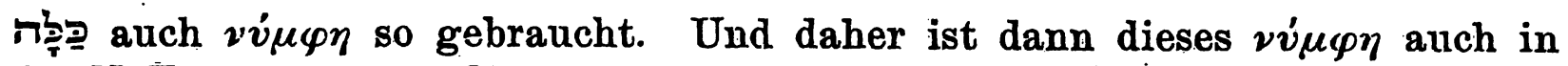
das N. T. gekommen. [Doch vgl. über hebraismen in der griech. bibel Thumb in Ilberg-Gerths N. jahrbiüchern 17 (1906) s. 252 ff.].

1) Freilich ist zu erinnern, dass nach ausweis der griech. wörterbücher vvós nur noch poetisch war; als regelrechte prosawidergabe des deutschen 'schwiegertochter' geben die deutsch-griechischen wörterbücher von Pape

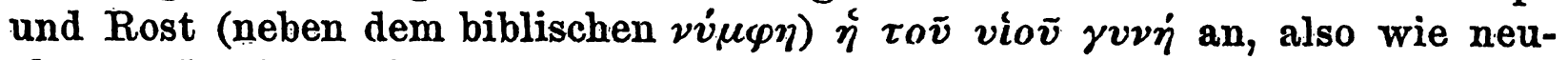

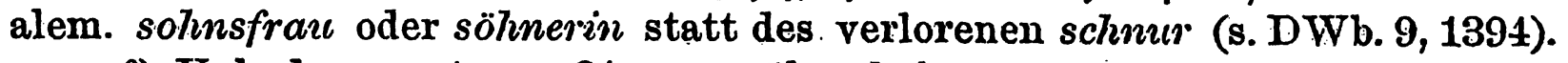

2) Vgl. dazu unten s. 31, anm. über krimgot. scłuos. 
drei alten inschriften stammen aus dem gebiete der unteren Donau. Danach hat schon v. Domaszewski mit wahrscheinlichkeit als den gebenden germanischen stamm die Goten bezeichnet. Er wies (N. Heidelberger jahrb. 3, 197) auf die vernichtenden niederlagen der Goten unter Claudius (269) hin, nach denen die gefangenen Goten in das römische heer eingereiht oder zu colonen des limes barbarus gemacht wurden. Durch diese elemente konnte der lat. heeressprache zunächst dieser gegenden das lehnwort brutis zugeführt werden. Zur erklärung der form knüpft Gundermann s. 245 an den got. nominativ brîps an und meint, dass die Römer die beiden spiranten $\not b+s$ nicht hätten ohne zwischenvocal sprechen können, indem er zugleich auf die beginnende assibilierung: von lat. $t i \mathrm{zu} z i$ hinweist. Dass das $i$. der alte themavocal sei, weist er ab. Denn dann würde einem vorwulfilanischen got. *brudis vielmehr lat. brudis, griech. $\beta \rho o v ́ \delta \iota s$ entsprechen müssen. Demgegenüber will $R$. Loewe die entlehnung nicht aus dem. gotischen, sondern aus dem westgermanischen geschehen sein lassen, welches damals die form des nom. als brüdiz geboten haben könnte. Aber die drei alten inschriften weisen durch ibre provenienz doch eher in die nähe der Goten. Und wenn die eine der inschriften aus Teurnia (Lurnfeld a.d. Drau in Kärnten) stammt, so liegt doch auch dies der sphäre der Goten näher als der Westgermanen. Wäre latein. brutis ein westgerm. lehnwort, so würden wir es am frühesten in inschriften aus den Rhein- und Neckargegenden zu erwarten haben, woselbst es aber vollständig fehlt. Und die schwierigkeit, dass westgerm. brüdi- im lateinischen doch auch nur als brudis zu. erwarten wäre, hat Loewe nicht weggeschafft. Ich meine, es muss aus geographischen gründen bei der entlehnung aus dem gotischen bleiben, - und auch aus sprachlichen. Denn gerade das sonst sehr merkwürdige lateinische $t$, griech. $\tau$ des lehnwortes lässt sich nur aus dem gotischen erklären. Allerdings nur unter der voraussetzung, dass das wort got. den stamm briupi- und nicht brüdi- gehabt hat. . Das ist aber höchst wahrscheinlich. Denn erstens gibt das westgermanische brüdi- uns nicht das mindeste recht, ohne weiteres auch ein got. br $\bar{\imath} d i$ zu erschliessen. In den worten auf idg. -ti-suffix fand germ. grammatischer wechsel statt, der dann verschieden ausgeglichen 
werden konnte. Solche verschiedenheiten finden sich sogar innerhalb des westgermanischen selbst. So z. b. niederfränkisch varth und ahd. Ludwigslied 38 hinavarth gegen sonstiges westg. fard, ahd. fart (vgl. auch Ahd.gr. $\left.\$ 163 a^{\prime} .6\right)$. Das gotische aber hat sehr häufig den grammatischen wechsel im nomen entgegengesetzt ausgeglichen, wie das westgermanische. Vgl. z. b. tagr gegenüber w.estgerm. tahar, ahd. zahar und suffix-ti in westgerm. giburd, aber got. gabaurps, g. gobaurp̈bais (ebenso das im westg. fehlende gataurps 'zerstörung', dat. gataurpai). Und wie dem westgerm. naudi- (ags. níed, ahd. nôt) ein got. naups, flect. naupai, naupim (daneben aber compos. naudibandi etc.) gegenübersteht, so kann auch dem westgerm. brüdi- ein got. brûps, gen. brûpais entsprochen haben. Einen positiven beweis dafür entnehme ich daraus, dass im gotischen in diesem worte niemals auslautend $\dot{d}$ erscheint. Wir haben es einmal im simplex acc. sg. brup und siebenmal im comp. brupfaps. Das letztere erscheint Mt. 9, 15 und Mc. 2, 19 zweimal im nom. brupfaps und zweimal im gen. brupfadis. Dass aber auch im ev. Lucas $(5,34.35)$, wo sonst auslautendes $\dot{d}$ für unechtes $\dot{p}$ so häufig ist, alle drei male die form brup-erscheint, das lässt sich nur daraus erklären, dass der stamm brîp- im gotischen ein echtes $\not p$ hatte. Nicht nur stehen in der umgebung: massenhaft $d$ für auslautend unechtes $\not b$ (Luc. 5, 30 matjid, drigkid; 34 magud, 36 lagjid, aftaurnid), sondern neben dem gen. brupbfadis erscheint der zweimalige nominativ als bruppfads! Also das kurzsilbige faps, welches unechtes $\not b$ hat, ist beide male fads geschrieben, während das langsilbige brup stets das $\not p$ bewahrt, trotzdem im Lucas grade nach langem vocal das auslautende $d$ herscht! (vgl. Got. gr. § 74, anm. 1). Und einen zweiten beweis für got. echtes $\not p$ in $b r u p s s$ gibt uns eben das $t$ in lat. brutis. Denn es ist bekannt, dass got. $\not{b}$. in lat. schreibung zwar oft durch th, aber auch oft durch $t$ widergegeben wird; vgl. z. b. Wrede, Sprache d. Ostgoten s. 170. Die schreibung th ist für die Römer eine gelehrte. . Für die harte spirans griech. $\vartheta$ und germ. $\not p$, wo sie dieselbe akustisch übernalumen, sprachen sie ihr $t$. Noch jetzt sind bei lehnworten in den romanischen sprachen die vertreter des germ. $\not b$ mit $t$ zusammengefallen. Somit beweist das $t$ in lat. brutis, dass got. $b r u p s$ ein echtes $\not{b}$ hatte, und so ist die entlehnung 
aus dem gotischen die sachlich und sprachlich einzig mögliche. Wir dürfen nun aber noch weiter gehen und aus brutis den schluss ziehen, dass hundert jahre vor Ulfilas das gotische den nom. noch als brüpis sprach, dass also die synkope des nominativvocals erst um 300 im gotischen eingetreten sein wird. Das hat auch gar nichts unwahrscheinliches, da das dem gotischen nächststehende urnordische die endvocale noch wesentlich länger behielt und auch das westgermanische, welches nach kurzer silbe noch um 900 das $i$ in wini, heti, gripi festhielt, wird zur zeit des Ulfilas noch brūdi(z) im nomin. gehabt haben. Gar so lange vor Ulfilas können wir also so wie so den schwund der endvocale für das gotische nicht ansetzen.

Ist so das lat. brutis formell ergebnisreich, so kommt für die bedeutungsgeschichte in betracht, dass in dem kreise dieses lehnworts nun allerdings die bedeutung 'schwiegertochter' auftritt. Das ist ohne zweifel der fall in dem späteren bruta. Diese form erscheint in lateinischen glossaren des 8. 9. jahrhunderts als erklärung des lat. nurus, welches also dem verfasser dieser glossare nicht mehr geläufig war. Am frühesten (mitte des 8.jh.'s) belegt ist die glosse nurus bruta in dem der keronischen glossensippe zu grunde liegenden lat.-lateinischen glossar, s. Ahd. gl. 1, 216, 20. 21, vertreten durch K, Ra und R.1)

1) In diesem von Gundermann a. a. o. nicht erwähnten ältesten beleg findet sich nun auch eine merkwürdige althochdeutsche glossierung. Das glossar $\mathrm{R}$ bietet zwar nur die lateinische glosse nurus, bruta; in $\mathrm{K}$ und $\mathrm{Ra}$ aber steht dazu die deutsche glosse proaton $\mathrm{K}$, proatun $\mathrm{Ra}$, die ich bei Kögel nicht besprochen finde. Man würde darin oblique casus eines sw. f. bruota zu sehen haben, das man mit brût nicht zusammenbringen würde, wenn nicht in einer Münchner hs. (12.jh.; Clm 13002) der glossae Salomonis nurus mit uxor filii vel br:ǒt erklärrt wäre, s. Gl. 4, 82, 1. 2; eine andere hs. (Clm 17152) hat nurus mit snûr übersetzt, drei weitere exemplare des glossars (Cod. Zwetl. 1, Cod. mon. sctae crucis 17, alter druck; vgl. Gl. 4, 152, 44, anm.) haben nur die lateinische glossierung nurus vel bruta. In dem brǒt von Clm 13002 würde man nun nach der überwiegenden schreibung der hs. zunächst ein zu jenem proatun von KRa im stammvocal auffällig stimmendes, aber starkes f. bruot sehen können, wenn nicht die bedeutung des zweiten $\check{o}$ in der hs. schwankte. Und da bald darauf (Gl. 4, 89, 21) gerade brǒtigoum erscheint, so ist es doch nicht zweifelhaft, dass brǒt hier als brût zu lesen ist. Daher muss man das proatun in KRa doch wol einer confusion der originalübersetzung zuschreiben und urteilen, dass eigentlich prut als übersetzung von nurus, bruta gemeint war. Wir 
Aus einer Erfurter hs. des 9. jh.'s hat G. Loewe die glosse nachgewiesen ${ }^{1}$ ) und man wird urteilen dürfen, dass die entstehung der lat. glosse wenigstens dem anfang des 8. oder dem 7. jahrhundert zuzuschreiben ist: wahrścheinlich rückt sie aber noch weiter zurück. Wie ist nun aber die brücke zu schlagen von diesem bruta 'schwiegertochter' der glossare $\mathrm{zu}$ dem brutis der soldateninschriften des 3.4. jh.'s und dem mgriech.

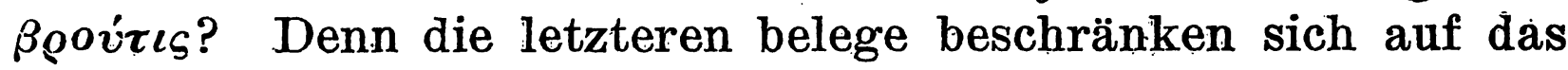
untere Donaugebiet und die. Balkanhalbinsel, während die glossen uns in das hochdeutsche gebiet und hinsichtlich der entstehung der lateinischen grundlage des keronischen glossars doch wol in den gallischen kreis führen. Auf dieses alte lateinische gloșar gehen sicherlich die übrigen späteren glossenbelege von nuirus bruta direct oder indirect zurück. Wir würden dann im westlichen culturkreise nur mit einem einzigen isolierten zeugnisse für bruta $\mathrm{zu}$ rechnen haben. Der lebendigen mlat. literatursprache des westens gehört dieses bruta nicht an: es muss also doch wol aus dem osten irgendwie durch die lateinische heeressprache dahin verschleppt sein, ohne weitere nachkommenschaft zu hinterlassen. Die östliche heimat wird schon durch die auf das gotische $b r \bar{u} \not p i$ - hinweisende form mit $t$ zweifellos bewiesen. ${ }^{2}$ )

Es fragt sich nun nach der ursprünglichen bedeutung dieses

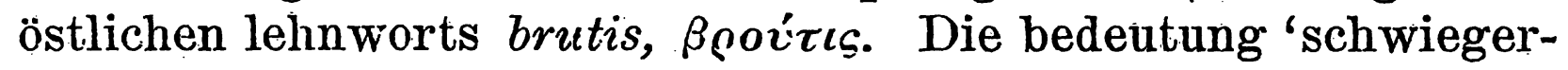

hätten also in diesen ahd. glossierungen die einzigen belege der bedeutung 'schwiegertochter' füx brût aus dem lebendigen und zụsammenhạngenden westgerm. sprachgebiete.

1) Vgl. Gundermann s. 240 und Goetz, Corpus gloss. lat. 5, 314, 32 (Gloss. Amplon. II saec. 9).

2) Weniger isoliert würde das westliche bruta dastehen, wenn es erlaübt wäre, das nordfranzösische bruy, bru, welches afranz. und noch jetzt nfranz. in der bedeutung 'schwiegertochter' vorliegt, darauf zurückzuführen. Das ist zwar früher mehrfach geschehen (vgl. z. b. J.Grimm, DWb. 2, 330; W. Deecke, Die deutschen verwautschaftsnamen, 1870, s. 164), lässt sich aber nicht aufrecht erhalten, da dann das afranz. wort notwendig zweișilbig sein müisste. So führt man denn franz. brı allgemein anf directe entlehnung aus niederfränk. br:îd żurück (s. G. Körting, Lat.-roman. wörterb.2 s. 170). Ebenso hait ein anderer romanischer dialekt, der rätoromanische, aus dem benachbarten alemannischen $b r \hat{\imath} t$ sein brit, breit etc: in der bedeutung 'schwiegertochter' entlehnt. Vgl. dazu E. Tappolet, Die roman. verwantschaftsnamen (Strassburg 1895) s. $130 \mathrm{f}$. 
tochter' liegt hier nicht zweifellos vor. Die belege des griech.

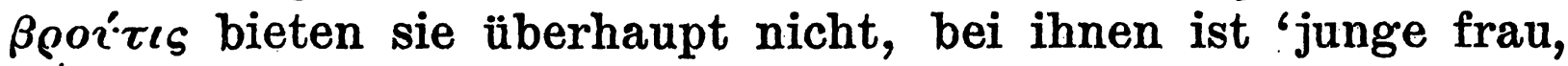
verheiratete frau' die vorwiegende bedeutung. Für die drei lat. inschriften hat allerdings v. Domaszewski die bedeutung 'schwiegertochter' angenommen, indem er von der landläufigen übersetzung des got. $b r \hat{u} p s$ ausgieng. Dass dieses argument nicht sicher ist, haben wir oben beim gotischen gesehen. Auch ist das genaue verwantschaftsverhältnis der unter brutis $\mathrm{zu}$ verstehenden jungen frauen aus dem zusammenhange dieser inschriften nicht sicher zu erkennen. Immerhin ist die bedeutung 'schwiegertochter' als möglich, teilweise sogar als wahrscheinlich anzunehmen und das westliche bruta, nurus kann als stütze dienen. Gundermann s. 246 hat die frage aufgeworfen, aus welchem grunde die römische heeressprache von den Goten das wort brutis aufgenommen habe, ohne darauf eine bestimmte antwort $\mathrm{zu}$ wissen. Keinesfalls aber darf die antwort die sein, welche R. Loewe a.a. 0.278 f. gibt. Im 10. jh. bei Suidas ist $\beta \rho o v ́ \tau \iota \delta \varepsilon \varsigma$ mit $\Sigma^{\prime} \beta v \lambda \lambda \alpha \iota$ oder $\pi \rho o \varphi \eta ́ \tau \iota \delta \varepsilon \varsigma$ erklärt. Loewe meint deshalb, die germanische frau in ihrer eigenschaft als seherin sei von den soldaten als brutis bezeichnet

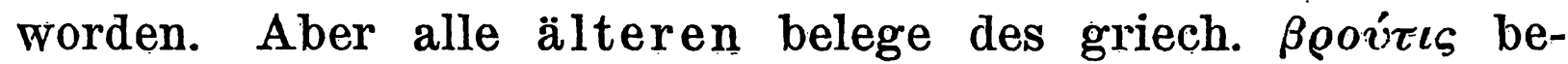
deuten einfach 'junge frau' ${ }^{\prime}$ ) und auch die lat. inschriften besagen dasselbe, oder das daraus abgeleitete 'schwiegertochter'. Für seherin würden die Goten wol ein anderes wort gebraucht haben, als grade br ûpis, dessen bedeutungscentrum doch sicher 'das weib in seiner geschlechtlichen function' gewesen ist.

Vielmehr scheint mir die tatsache der beachtung wert, dass die bedeutung 'schwiegertochter' unabhängig in drei verschiedenen lateinisch-romanischen entlehnungsgebieten sich festgesetzt hat: im nordfranz., rätoromanischen und im Unterdonaulande, obwol die gebenden germanischen dialekte das wort in dieser bedeutung nicht gebrauchen: das niederfränkische und das alemannische sicher nicht ${ }^{2}$ ), und auch das gotische

1) Aus welcher trïben quelle Suidas seine angabe geschöpft hat, könuen wir nicht wissen: jedenfalls liegt hier aber ein misverständnis vor, denn im 10.jh. war Beoviıs im griechischen schon nicht melur allgemein gebräuchlich, wie Gundermann s. $2 \pm 4$ feststellt.

2) Von den oben s. 41, aum. 1 erwähnteu ahd. glossierungen abgesehen, 
zum mindesten nur occasionell. Es scheint also die aus 'junge frau' sich jederzeit ungezwungen entwickelnde bedeutung 'schwiegertochter' gewesen zu sein, welche das wort für diese verschiedenen romanischen gebiete als wünschenswerten erwerb erscheinen liess und seine festsetzung begünstigte. Voraussetzung war, dass diese gebiete das erbwort nurus nicht mehr kannten und einen ersatz dafür brauchen konnten. Und in der tat ist dieser verlust auf vielen romanischen gebieten eingetreten, ebenso wie im spätgriech. vvós verloren war (oben s.38, anm. 1), und wie im deutschen seit dem 16. jh. das alte schnur allgemein untergegangen ist. ${ }^{1}$ ) Dabei wird diêsen entlehnungsgebieten das germanische wort zuerst in seiner eigentlichen bedeutung bekannt geworden sein: dafür beweist einmal $\beta \rho o c^{\prime} \tau \iota s$ 'junge frau' und als schlagende parallele das frz. bru, welches nach Tappolet s. 131 in gewissen nordfranzösischen dialekten noch 'nouvelle mariée' bedeutet. Aber diese bedeutung haftete nicht: es blieb nur die daraus abgeleitete bedeutung 'schwiegertochter', welche eine lücke ausfüllte.

Dass nun das ursprüngliche bedeutungscentrum von brîd in der geschlechtlichen function lag; das lehren ganz besonders die westgerm. sprachen. Am deutlichsten sprechen hier die älteren deutschen belege vor der nhd. verschiebung des begriffes. Das althochdeutsche ist nicht allzu ergiebig, da hier meist kirchliche übersetzungsliteratur vorliegt, in der brût als widergabe des biblischen sponsa erscheint ${ }^{2}$ ), welches, wie oben gezeigt ist, im wesentlichen dem ne. bride (braut am hochzeitsfeste und neuvermählte) entspricht. Die belege aus $O$. haben wir schon besprochen. Im N. Marc. Capella tritt brût auf als

die in ihrer vereinzelung wol durch das lat. bruta herbeigezogen sind und bestenfalls die principielle möglichkeit einer solchen bedeutungsentwicklung auch für das ahd. dartun. - Aus neuerer zeit bringt W. Schoof (Die deutschen verwandtschaftsnamen, Zs. f. hochd. mundarten 1, 193 ff.) s. 274 aus dem Aargau die aualoge ïbertragung' die jung (junge frau) 'schwiegertochter' bei. Und umgekehrt s. 271 die anwendung von schnur, suns weib $=$ 'sponsa, nova nupta'.

1) Ueber beschränktes weiterleben in mundarten vgl. Schoof s. $272 \mathrm{f}$.

3) Wenn Graff 3, 293 aus einer Berner Prudentiushs. des 11.jh.'s neben mupta (Gl. 2, 527,56) auch prut als übersetzung des lat. pact (pactam Gl. 2, 527, $49=$ Prud. contr. Symm. 1, 258, pactae G1. 2, 528, $17=$ Prud. c. S. 1, 471), so ergibt sich, dass hier von Prud. pacta poetisch für 'die 
jungvermählte ed. Piper 1,690, 7 (des cómenes únde dero brûte), sowie als noch jungfräuliche braut am hochzeitsfeste 82S, 12 . 842,26 , wo im latein. virgo steht. Diese ausdehnung des begriffs brût von der neuvermählten auf den letzten tag ihres jungfräulichen standes am hochzeitsfeste ist eine charakteristische erscheinung, welche den ausgangspunkt für die nhd. umdeutung gebildet hat, aber keinesfalls dazu führen darf, für das germ. wort vom grundbegriff 'jungfrau, integra' (vgl. Wiedemann s. 206. 208) auszugehen. Dass vielmehr die geschlechtsfunction das wesentliche ist und dass der hochzeitstag nur durch eine art vorausnahme schon zum zustand des neuvermähltseins hinzugezogen wird, dafür geben aussergermanische sprachen reichlich belege. Am schlagendsten ist hier die vergleichung des neufranz. sprachgebrauchs. Sachs-Villatte, Encycl. wb. 2, 307 gibt für nhd. braut folgende nfrz. entsprechungen an: = 'verlobte': fiancée, future; = 'braut am hochzeitstage': épousée, (nouvelle) mariée; und für nhd. 'bräutigam': fiancé, futur époux; aber am hochzeitstage: marié. Also nhd. 'braut' und 'bräutigam' werden am hochzeitstage nfranz, durchaus schon mit dem worte bezeichnet, welches den stand der ehelichen verbindung bezeichnet, grade wie bei brût und brûtigomo im ahd. Und das griech. ví $\mu \varphi \eta$ wie das litauische martì (Wiedemann s. 208) haben denselben bedeutungsumfang. Wenn aber' Wiedemann nun sagt: 'wir dürfen annehmen, dass sowol das litauische als auch das germanische wort ursprünglich »mannbares weib « ohne rücksicht auf unberührtheit bedeutet hat; erst mit der geburt des ersten kindes beginnt ein neuer abschnitt im leben des weibes', - so widerspricht dieser umgrenzung doch der westgermanische sprachgebrauch. Dass die geburt des ersten kindes nicht endpunkt des begriffes brût war, zeigt schon das ahd. Hildebrandslied, in dem Hildebrant die prût und das barn unwahsan verlassen hat. Und dass nicht nur mannbarkeit, sondern die mit dem hochzeitstage beginnende geschlechtliche activität das kennzeichen der brût

vermählte' (im nhd. sinne) gebraucht isț, die übersetzung mit ahd. prût also nichts für die nhd. bedeutung: beweist. Auch Vergil Aen. 10, 79 braucht dieses wort, welches in lateinischer prosa allerdings 'verlobte' hiess, schon übertragen $=$ ahd. brût. In Gl. 2, 715, 56 (cod. Paris.) ist dieses Vergilische pactas mit gemâlda gegeben. 
war, lehren uns ganz besonders die belege aus der reichen mhd. literatur, wofür die mhd. wörterbücher s. v. genügendes material beibringen. Sehr lehrreich ist die von Müller ausgeschriebene stelle aus Heinrich v. Freiberg, Tristan 867: ouch gienc Isôt, Tristantes trût, die mit dem namen was ein brût und noch der werke was ein maget etc., wo der gegensatz zwischen brût und maget scharf pointiert erscheint. Ebenso MSH 3, 418a würde $\hat{u} z$ der meide niht ein brût und dazù parallel in derselben strophe gleichbedeutend wïrde $\hat{u} z$ der meide niht ein wîp. Und die stelle in Hartmanns Gregorius 385 ff. si gedâhte: swîge ich stille, sô ergât. des tiuvels wille und wirde mînes bruoder brût, zeigt deutlich, dass die geschlechtliche vereinigung nach mhd. sprachgefühl das wesentliche moment des begriffes $b r \hat{u} t$ bildete.

Hierfür zeügt auch das abgeleitete verbum mhd. briuten, dessen grundbedeutung ist 'concumbere cum aliqua', 'ein weib durch das beiliegen zur brût machen', 'futuere, stuprare'. Das wort hat eine besonders reiche entwicklung auf niederländischem und niederdeutschem gebiete genommen, vgl. für das mndl. Verwijs-Verdam, Wb.1, 1463: bruden 'eene vrouw beslapen, gemeenschap met haar oefenen'. Ebenso mnd. brüden Schiller-Lübben 1,$434 ; 6,88$. Während das verb im nhd. verloren ist (Grimms belege im DWb. 2, 333 unter brauten, bräuten reichen knapp bis ins 16. jh.), so finden wir nd. noch in Laurembergs scherzgedicht 4, 686 brïd dine möme 'stupra matrem tuam'. Ich habe in meinem glossar zu Lauremberg (Neudrucke des 16. 17. jh.'s, Halle 1879) s. 89 dargelegt, wie aus dieser grundbedeutung sich das neund. brüden, brüen zu der bedeutung 'vexare', jem. belästigen, plagen, necken, entwickelt hat, in welcher allein es in den neund. maa. noch heute lebendig ist, während oberd. sich das von Hildebrand, DWb. 4, 1, 2340 ausführlich behandelte geheien in ähnlicher weise gewandelt hat. Genau wie im niederdeutschen hat sich im neuniederländischen das verbum bruien (älter bruiden) gestaltet. Hierüber haben wir im Woordenboek der Nederl. taal bd. 3, 1 (von Muller und Kluijver) s. $1619 \mathrm{ff}$. ausführliche nachweisungen. Das nndl. bruien (bruiden) bedeutet 1) 'eine frau beschlafen'. Dies wird aber im 17. jh. schon als vulgär empfunden und verschwindet dann. 2) 'vexare', im 17. 18. jahrhundert sehr gewöhnlich, jetzt aber im veralten. 
Das mhd. briuten, mnd. mndl. brîden scheint dem südwestgermanischen eigentümlich zu sein: weder altn. *brýda noch ags. *brydan finden sich. Und auch hier ist der älteste beleg erst aus N.'s psalmen (Graff 3, 294). Es könnte also dieses verbum eine jüngere neubildung des continental-deutschen sprachgebiets sein, welches mit seiner besonders in Niederdeutschland mehr nach der obscönen seite gerichteten bedeutungsentwicklung für uns hier dann nur insoweit in betracht kommt, als auch dadurch für das zu grunde liegende ahd. alts. brît, brûd als centrale bedeutung gesichert wird 'quae concumbit cum aliquo'.

Wenn also im altdeutschen brût die bettgenossin eines mannes ist, eine bedeutung, aus der auch die oben s. 6 ff. von uns festgestellte weitere anwendungsweise der alts. und ags. dichtung sehr wol zu verstehen ist, so ist doch nach zwei seiten hin noch die grundbedeutung näher zu definieren. Einmal ist hervorzuheben, dass in allen lebenden neugermanischen schriftsprachen das wort $b r \bar{u} d$, braut sich heute nur auf das legitime verhältnis bezieht, nhd. braut ist die verlobte, nndl.bruid, neuskand. brud die braut unmittelbar vor der hochzeit, ne. bride die jungvermählte mit einschluss des hochzeitstages. Aber diese beschränkung liegt nicht in den älteren sprachen vor. Quae cum aliquo concumbit, wird nach den im princip monogamischen verhältnissen des deutschen altertums in den meisten fällen das legitime weib sein. Jedoch kann auch jedes illegitime verhältnis, ja jedes weib, zu dem ein mann in gelegentliche geschlechtliche beziehungen tritt, mit dem worte bezeichnet werden. Wenn z. b. mndl. (aus der Minnen Loep bei Verwijs-Verdam, Wb. 1,1469) von der biblischen Susanna gesagt wird: Suzanna soude werden hoirre beyder bruut ende soude doen al hoer ghenuegen, so bezieht sich dieses, ebenso wie die vorhin citierte stelle Hartm. Gregor. 385 auf den einmaligen fall, während Kudr. 1029, 4 Hartmuots worte wer hienge mich darumbe, ob ich iuch mir gewïnne ze einer briute?, wie auch Kudruns antwort 1030, 4 bestätigt, sich auf das verhältnis der kebese beziehen. Aber es ist durchaus falsch, wenn wörterbücher die bedeutungen danach gliedern. So definiert das Mndl. wb. 1,1469 bruut 1) De verloofde zoowel als de jonggehuwde vrouw '), 2) bijslapster; ähnlich trennt Lexer die beiden

1) De verloofde ist falsch und nur durch die daselbst citierten deutschen 
bedeutungen. Denn brût ist hinsichtlich des rechtlichen verhältnisses ganz indifferent, es ist an sich weder mhd. vriedel, noch leebese, noch êlîch wîp (êleone), sondern alles gleichmässig in einem, es bezieht sich rein auf das śexuelle, physiologische verhältnis zum manne, es ist das weib, welches dem manne beiliegt oder eben im begriff ist ihm beizuliegen. Insofern ist am rationellsten Müllers bedeutungsentwicklung im Mhd. wb. 1, 273: '1) im allgemeinen bezeichnet dieses wort eine weibsperson, die einem manne unlängst beigelegèn hat, oder nächstens beiliegen soll; 2) daher heisst brût a) die rechtmässige gemahlin kurz vor oder bald nach der vermählung, b) die beischläferin, das kebsweib'.

Zweitens haben wir nun noch nach der zeitlichen begrenzung des seinem sachlichen gehalte nach festgestellten begriffes von germ. brûd zu fragen. Insbesondere wird die frage jetzt zu beantworten sein, von der wir ausgegangen sind, wie sich die anwendung des alts. ags. brûd, brýd auf ehefrauen im hohen lebensalter, also die bezeichnung der hundertjährigen Sara als Abrahames brýd zur gründbedeutung stellt. Und da wird denn doch wol die antwort die sein müssen, dass wir hierin eine erweiterung des gebrauchs seitens der altwestgerm. alliterationspoesie $\mathrm{zu}$ sehen haben. Denn die übereinstimmung der germ. sprachen weist auf die zeitliche begrenzung von brûd auf das weib jugendlichen alters hin. Im bereiche des hochdeutschen sprachgebiets ist mir kein fall bekannt, wo brût zeitlich darüber hinausreichte, auch die mndi. belege stimmen dazu. Auch aus dem kreise des got. brîps mit seinem mlat. und mgriech. ableger haben wir ebenfalls nur die beziehung auf ein junges weib kennen gelernt. Und auf dem englischen sprachgebiete selbst zeigt das ne. bride, abgesehen davon dass es auf das legitime verhälthis eingeengt ist, übereinstimmend mit den übrigen germ. sprachen die beschränkung: auf das junge weib. Aus dem ags, sind mir wenigstens in der prosa keine sicheren beispiele für zeitlich unbeschränkte geltung von brýd aufgestossen. Aus der me. zeit zeigen die ziemlich zahlreichen belege von brud, bruid, brid; burd u.s.w. bei Mätzner, Sprachpr. 2, 1,350 f. neben der ne. bedeutung noch

wörterbücher verursacht. Wie die belêge ausweisen, ist die bedeutung genau dieselbe wie im mhd. 
eine andere erweiterung des gebrauchs in der poetischen sprache. Es wird das wort da nämlich absolut gebraucht für 'junges weib, mädchen, jungfrau', ohne rücksicht auf das verhältnis zu einem manne, z. b. Denna com he of his closet, with mony cler burdez (Gawayn) oder von Maria: Anes maidenes sune iboren wes in Beðleem of bezste alre burden (Layamon). Dass hier nur poetische übertragung vorliegt, ist klar. Für die anwendung der ags. poesie auf ältere frauen habe ich aber keine me. beispiele gefunden.

Auf sächsischem gebiete gibt uns dagegen das wb. von Schiller-Lübben unter brut und brudegan $(1,438.434)$ bemerkenswerte belege des erweiterten gebrauchs. Z. b. in einem Oldenburger manuscript (beschreibung der messe) wird vom priester gesagt: darna biddet he vor den paves unde vor den keiser, der na nomet he sine brut. Also hier wird die kaiserin ohne beziehung auf das lebensalter in einem prosadenkmale des keisers brît genannt. 'In der mnd. Melusina heisst diese noch brût, nachdem sie Reymund zehn söhne geboren, dieser ihr brudegam.' Es scheint also auf sächsischem gebiete der in der alts. poesie uns entgegentretende gebrauch tiefer und fester in die lebende sprache eingedrungen zu sein.

Weit enger ist die anwendungsweise des wortes in der altn. prosaliteratur. Daselbst gilt brúdr nicht mehr von der jungen frau, sondern nur von der braut während der hochzeitsfeierlichkeiten, zu denen aber auch schon die reise zum hause des bräutigams, wo die hochzeit gehalten würde, gehört: die brúđferð oder brúðför, s. Cleasby-Vigfusson s. 83. 84. Mit dem antritt dieser fahrt wurde die bisherige festarmey zur brídr, welcher name ihr also ev. eine reihe von tagen zukam. Die neunordischen sprachen, schwedisch, dänisch, nisl. beschränken dagegen den namen brud auf den einzigen tag der hochzeit; vgl. z. b. Helms, Wb. d. dänischen spr. 1,61 s. v. Brud 'braut, an dem tage ihrer hochzeit'; im sinne des deutschen 'braut' als verlobte kommt dän. brud 'nur selten, und dann poetisch' vor. Hier haben wir also eine vielleicht unter deutschem einfluss stehende poetische erweiterung des begriffs vor uns.

Wie ist nun diese von den übrigen altgerm. sprachen abweichende anwendung von altn. brüđr, dän. schwed. brud aufzufassen, indem die 'junge frau' hier vom bedeutungsumfange 
ganz ausgeschlossen ist? Wer vom nhd. sprachgefühl und der etymologischen verlobungstheorie ausgeht, könnte meinen, dass das skandinavische brud etwas ursprünglicheres darin zeige, dass es nur den endtermin der brautzeit im nhd. sinne beibehalten habe. Dass es sich aber umgekehrt verhält, dass vielmehr das nord. brud, welches nur noch den anfangstermin der altgermanischen brautzeit, die hochzeitsfeier, festhält, das hauptgebiet seines alten bedeutungsumfangs eingebüsst hat, ist ganz unzweifelhaft. Merkwürdig ist nur, dass schon die altisl. prosadenkmäler diese verengung zeigen. Aber einen ganz ähnlichen vorgang, der im geschichtlichen verlauf zu beobachten ist, zeigt die verschiebung des begriffs im neuniederländischen, womit zugleich ein zwischenglied zum noch weitergehenden wandel des nhd. gegeben wird.

Im neuniederl. bedeutet nach der eingehenden behandlung im Neuniederl. wb. 3, 1 (v. Mùller en Klụyver, 1902), s. 1620 ff. ndl. bruid nach der jetzt geltenden auffassung: 'ondertrouwde vrouw' d.h. durch offizielle anmeldung bei der behörde öffentlich verlobte frau. 'Strikt genomen derhalve alleen toepasselijk zoolang het paar onder de geboden staat (so lange das öffentliche aufgebot währt), van de aanteekening tot aan de huwelijksvoltrekking; gemeenlijk echter ook nog gedurende den trouwdag, doch dernaa niet meer, aan de jonggehuwde gegeven.' Hier ist also die bedeutungsverschiebung nach vorn nur auf eine ganz bestimmte kurze zeit vor dem hochzeitstage vollzogen (vgl. die zeit der altisl. brúðför). Aber lange nicht so weit wie im nhd. Denn die nhd. braut heisst im niederl. verloofde, bis das aufgebot angemeldet ist, dann erst wird sie bruid. Nur bei neuniederl. dichtern findet sich freierer gebrauch. Das Wb. fährt fort: 'Bij dichters ook wel eens, evenals in het nhd., in ruimere opvatting: verloofde'. ') Wichtig ist, dass im niederl. heute bruid nur noch bis zum hochzeitstage incl. reicht, wie im nhd. und altisl., während die ältere sprache grade umgekehrt erst vom hochzeitstage an rechnet. Dieser ältere gebrauch hat im niederl. bis ins 17. jh. gedauert. Im Woordenboek heisst es 2) 'Naer de oudere ruimere opvatting ook in toepassing op (jong)gehuwde vrouwen. Thans geheel verouderd.'

1) Vgl. die oben erwähnte gleiche übertragung bei neudänischen dichtern, und Gladstones misbrauch des ne. bride oben s. 30, anm. 
Die belege des $\mathrm{Wb}$. für diesen jetzt veralteten gebrauch sind aus Vondel, Hooft und Bredero. ${ }^{1)}$

Im altn. ist also diese verschiebung, welche das hauptgebiet der altgerm. bedeutung, die zeit der 'jungen frau' abtrennte und nur die hochzeit selbst für brüđr übrig liess, früher als in anderen germ. sprachen eingetreten: im 12. 13. jh., zur zeit der sagaschreibung, sehen wir die neue geltung schon durchgeführt. Demgegenüber beweisen aber die hier wie sonst älteres sprachgut bewahrenden altn. poetischen quellen, dass darin eine verengerung des alten begriffs liegt. Das material liegt bei Sveinbjörn Egilsson 85 und für die Edda vollständig vor in Gerings wörterbuch s. $133 \mathrm{f}$. (Die lieder der Edda hg. v. B. Symons und H. Gering, bd. 2, Halle 1903). Hier zeigt sich die altgermanische bedeutung noch im weitesten umfange. Wenn Gering als hauptbedeutung an die spitze stellt 'verlobtes oder jungvermähltes weib', so treffen beide definitionen die sache nicht ganz genau. Das 'verlobtes' ist dem nhd. gebrauch zu verdanken, welcher, wie schon hervorgehoben, so oft die lexicographen und etymologen irre geführt hat ${ }^{2}$ ); 'jungvermählt' aber ist zu eng, denn in der altn. poesie ist brúdr noch durchaus nicht auf das legitime verhältnis beschränkt, sondern bezieht sich wie im mhd. mndl. gleicherweise auch auf die aussereheliche geschlechtsgemeinschaft, weshalb Sv. Egilsson richtig neben 'sponsa, nympha, nova nupta, uxor' auch 'puella, amica, amasia' umschreibt. So übersetzt Gering selbst Vkv. 35 die stelle: at pú Teveljat kvọn Volundar, né brúbe minne at bana verper in seiner Eddaübersetzung $\mathrm{s.147}$ sehr richtig mit 'dass du Wölunds gattin nicht weh bereitest und meiner geliebten das leben nicht raubst'. Wenn Bopvildr hier Völunds brupr heisst, so ist diese eigenschaft durch einen notzuchtsakt begründet. Vorher aber Vkv. 19 hatte Völund mit den worten nú berr Bopvildr brübar minnar bauga raupa seine gattin Hervor brüpr genannt, welche er acht jahre besessen hatte. Und in HH. I, 44 pu brupr Grana ... vast 'du hast (als stute) mit dem hengst Grani unzucht getrieben' ist briupr

1) Ganz die gleiche bedeutungsumgrenzung gibt das Woordenb. für nndl. bruidegom $(3,1,1632)$.

2) Denn selbst in stellen wie Alv. $1 \mathrm{ff}$. und H. Hr. 32. 41 ist brúpr in allgemeinerem sinne zu verstehen: die zur brúpr bestimmte, die geliebte. 
sogar auf tierische verhältnisse angewant. Andrerseits ist auch hervorzuheben, dass in der Edda brúpr als gattin schlechthin, ohne beziehung auf jugendliches alter, angewant wird: vgl. jarla brúper 'die weiber der jarle' Gpr. I, 3; jqtna brüper, berserkja bripper 'weiber von riesen, von berserkern' Hrbl. 66. 99; fylkes brúper 'die weiber des königs' H. Hv. 3. Und so citiert denn Sveinb. Egils. s. 123 eiginbrúdr uxor (sína eiginbríði aus der Placidus drápa) als synonymum des prosaischen eigintiona. Daneben aber wird auch in der altn. poesie metonymisch brúdr für 'weib' im allgemeinen gebraucht, ohne beziehung auf das verhältnis zu einem manne. Hierin haben wir, wie oben im mittelenglischeñ, eine poetische übertragung zu sehen. Die beispiele aus der Edda stellt Gering zusammen. So Grip. 16 von Brynhild brüpr moela tekr, es vakkape vif or svefne. Hier ist brúpr synonym mit víf gebraucht. Oder Vsp. 22,4 de vas angan illrar brúpar '(die zauberei) war stets das vergnügen bösen weibes'.

Wir dürfen also auch den skandinavischen sprachzweig auf grund des ältesten poetisçhen gebrauchs für die allgemein germanische bedeutung des wortes brîd in anspruch nehmen, die ich als 'mulier quae cum viro concumbit' definiere. Die geschlechtlich active frau wird der sachlage nach im wesentlichen die frau des blühenden alters sein, so dass also das 'junge mit dem mann verkehrende weib' den' bevorzugten bedeutungsumfang darstellt. Aber auch alle anderen gebrauchsweisen leiten sich daraus unschwer ab. Besonders stark entwickelt ist infolge der monogamischen richtung des geschlechtsverkehrs die einengung auf das legitime verhältnis, so dass also die 'jung vermählte frau' eine sehr häufige bedeutung ist. Von da aus kann die in der alts. und ags. poesie besonders deutlich hervortretende anwendung auf die ehefrau ohne rücksicht auf das lebensalter sich ableiten, andrerseits unter stärkerer betonung des jugendlichen alters die beschränkung auf die erste zeit der jungen ehe, wie sie noch heute das ne. bride bewahrt hat. Da aber der hochzeitstag und das hochzeitsfest wie bei andern völkern (griech. $v \dot{u} \mu \varphi \eta$, franz. nouvelle mariée) schon als erster tag der jungen gemeinschaft in den wortbegriff einbezogen wurde, so konnte dieser tag und dieses so wichtige fest als bedeutsamste seite des wortumfangs 
specialisiert werden, unter abstossung der anwendung auf die zeit der jungen ehe. So in den skandinavischen sprachen seit der sagazeit. So auch im neuniederländischen nach dem 17. jh., indem dabei der brautname auch noch auf die zeit des öffentlichen aufgebots erstreckt wurde. So endlich mit der vollkommensten verschiebung im nhd., welches allein die bezeichnung 'braut' und 'bräutigam' auf die ganze zeit des verlobtseins ausgedehnt hat, wobei jedoch lange das sprachgefühl dafür wach blieb, dass das hochzeitsfest den ausgangspunkt für diese namen bilde. Noch Adelung in seinem wörterbuche (2. aufl.) 1, 1168. 1170 definiert braut: 'eine verlobte person weiblichen geschlechts, und in engerer bedeutung, eine solche person am tage der hochzeit', bräutigam: 'eine verlobte person männlichen geschlechts, besonders am tage der hochzeit'.

Es wäre nun von interesse festzustellen, wann und wo die nhd. bedeutungsverschiebung eingetreten ist; dass also brant nicht nur auf das hochzeitsfest bezogen, sondern auch unabhängig davon auf die verlobte angewant wurde. Durch diese verschiebung ist es jetzt so weit gekommen, dass in der heutigen hochsprache das bedeutungscentrum des nhd. braut gradezu im begriffe der jungfräulichkeit, der unberührtheit der verlobten liegt, so dass das wort einen hohen und edlen klang hat, ganz abweichend von dem von uns festgestellten altgermanischen bedeutungscentrum. Als vermittlung zu diesem heutigen gebrauch hin wird man erwarten können anwendungsweisen, die gewissermassen proleptisch braut auch schon auf frühere zeitstufen beziehen, aber dabei doch immer in hinsicht auf die nachfolgende hochzeit gemeint sind. Eine solche vorausnahme ist es, wenn der Engländer seine verlobte intended bride u.s.w. nennt (oben s. 30, anm.), oder die vereinzelte poetische anwendung des wortes auf die verlobte bei neuniederländischen und dänischen dichtern. Das alles ruht noch auf dem alten bedeutungscentrum.

Aber für die feststellung dieses übergangs lassen unsere neueren wörterbücher uns sehr im stich, da die verfasser meist gar nicht bemerkt haben, worauf es ankommt, indem sie die nhd. bedeutung, durch das ahd. brût = sponsa verleitet, auch schon dem altdeutschen zuschreiben. Die belege .in Jacob Grimms artikeln brant und bräutigam im DWb.2,330 ff. be- 
ziehen sich sämmtlich auf die hochzeit, auch die aus Luthers bibelsprache: kein einziger bezeugt einwandsfrei die dem nhd. eigene neue bedeutung. Auch die grosse fülle der composita beweist lediglich für den ältern sprachgebrauch. Nur brautstand (s. 338) wäre für uns wertvoll: aber der älteste beleg Grimms ist aus Wagners Kindesmörderin. Wer also behaupten wollte, erst seit der classischen periode unserer dichtung habe braut die neue bedeutung 'verlobte' entwickelt, der könnte durch Grimms DWb. nịcht widerlegt werden. Aber das wäre doch nicht richtig. Bei durchsicht der literatur ergibt sich bald, dass dieser gebrauch älter ist. Gellert übt ihn '), und Meta in ihrer correspondenz mit Klopstock unterschreibt sich am 24. nov. 1752 'deine braut' und nennt Klopstock (an Giseke 27. nov. 1753) ihren 'liebenden bräutigam'.2) Hier liegt schon ganz das heutige bedeutungscentrum zu grunde.

Auch Steinbach, Vollst. deutsches wb. (1734) 1, 189 f. übersetzt braut mit sponsa. desponsa, desponsata, also 'verlobte' und in das 17. jh. führt uns sein citat aus Hofmanswaldau: Da ich das erste mahl das fremcle wort vernommen, Wo seufftzer worte sind, Algerth ist meine braut, während sein zweites dichtercitat aus Günther: Wer wollte bey der braut voll finsterer grillen sitzen mit der übersetzung: 'Quis vero rugosam frontem ad nuptias adferret' auch aus der alten bedeutung zu erklären wäre. - Bei Stieler, Teutscher sprachschatz (1691) s. 224 wird braut übersetzt: sponsa, desponsata, nova nupta und die belege: 'Einem eine braut versprechen despondere filiam alicui; Sich eine brant nchmen despondere sibi alicujus filiam' zeigen ebenfalls die neue bedeutung. . Und A. Gryphius nennt in der widmung vom Verliebten gespenst und dornrose das 'fïrstl. fräulein braut'.

Dagegen führt uns Josua Maaler, der in seinem Dict. germ. 1561 s. 77 braut mit Nupta, Sponsa, Nympha, Marita, Nova nupta übersetzt, einen sprachgebrauch vor, der durchaus noch im alten bedeutungscentrum wurzelt. Es ist deshalb bemerkenswert, dass schon vorher in Luthers schriften die anwendung von braut auf die verlobte deutlich vorhanden ist.

1) Z.b. Zärtliche schwestern 1,4: 'anstatt dass ich glaube, Julchen heute als meine braut $z u$ sehen'.

2) Briefe von und an $\mathrm{Kl}$. ed. Lappenberg; s. 114. 127. 
Die vorwiegende anwendung der worte braut und bräutigam bei Luther ist allerdings mit der hochzeit verknüpft. Aber es sind genügend beispiele nachweisbar, welche davon losgelöst nur die verlobten im nhd. sinne bezeichen. Reichliche belege für braut und bräutigan findet man im 10. teil der Lutherausgabe von Walch (Halle 1744), woselbst s. 693-977 die auf das sechste gebot bezüglichen schriften zusammen abgedruckt sind.1) Indem ich die zahlreichen beispiele des älteren gebrauchs ganz übergehe (hierher auch die Lutherbelege unter braut in Grimms DWb.), führe ich nur einige stellen an, aus denen die neue bedeutung bei Luther klar hervortritt. Walch 10,718 wenn mir meine braut stivbt, ehe ich sie heimhole, so darf ich nicht nehmen ihre schwester (vgl. s. 743); s.775 die verlobte und vertraute braut, die noch der bräutigam nicht erkannt (ähnlich s. 922); s. 926 der öffentlichen verlobten braut (vgl. 924); s. 933 gleichwie der pabst erlaubt und gebeut, 'dass eine ehefrau mag ihren mann aus dem kloster fordern, also sollte ers auch braut und bräutigam erlaubet und geboten haben, dass sie nicht von einander ins kloster liefen. Andere stellen s. 896. 923.931.

Aus der deutschen schriftsprache vor Luther sind mir bis jetzt sichere beispiele für braut $=$ 'verlobte' nicht begegnet. Ich habe daraufhin die 'Deutschen privatbriefe des Ma.' hg. von Georg Steinhausen (Berlin 1899) durchgesehen und darin aus dem 15. jh. das folgende material gefunden. $\mathrm{S} .45$ (no. 58 vom 23. febr. 1442). In einer hochzeitseinladung an seinen bruder spricht herzog Wilhelm von Sachsen von seiner braut, indem er sie zweimal unser liebin vertruweten gemaheln nennt, wobei das vertruweten wesentlich ist (vgl. oben s. 32, anm. 2), da das einfache gemahel auch schon 'gemahlin' bedeuten konnte, wie es im selben briefe daneben gebraucht wird. - S. 320 (no. 474 vom 21. aug. 1496). Herzogin Sidonie von Sachsen schreibt an ihren verlobten sohn Georg, dessen hochzeit um Martini sein soll, also ein vierteljahr vorher: got gebe dir und deiner gemahel gar vil glucks und.heiles, wo also für braut noch das einfache gemahel angewendet wird. Im selben briefe

1) Ich citiere der einfachheit halber nur nach dieser ausgabe, zumal der grössere teil der betreffenden schriften in der Weimarischen ausgabe noch nicht erschienen ist. 
neckt sie scherzend ihren sohn, der in der zerstreutheit einen brief verwechselt hat, man könne auf ihn jetzt das sprichwort anwenden: du ghest yn gedancken als eyn verlobte mayt. Also 'zerstreut wie eine braut' würden wir das ausdrücken. Dieselbe wendet dagegen s. 319 (no. 473) das wort braut an im zusammenhange mit dem hochzeitsfeste (geschenk an die braut bei der hochzeit). - Ebenso aúch sonst braut nur bei der hochzeit. So s. 295 (no. 434) zweimal in einem briefe von 1491. Und s. 198 (no. 288) hochzeitseinladung Albrechts von Brandenburg vom 6. febr. 1479: das das elich beiliegen sein wird uff sonntag Valentini ${ }^{1}$ ) . . unnd wirt die preut auff sambstag davor hieher komen. S. 157 (no. 224 vom 14. febr. 1476) schreibt derselbe an Ulrich von Württemberg, wünscht glück zur bevorstehenden hochzeit von dessen tochter: darzu euch und unser lieben swiger, auch euer tochter, der preut... got vil gluchs geb. Die beiden letzten stellen kann man schon als beispiele einer art von vorausnahme ansehen, indem hier vorher diejenige als 'braut' bezeichnet wird, welche auf der hochzeit diese rolle zu spielen hat.

Soweit ich bis jetzt urteilen kann, scheint mir der gebrauch von braut für 'verlobte' aus Ostmitteldeutschland zu stammen und von dorther in die schriftsprache seit Luther aufgenommen zu sein. In der ostmitteldeutschen mundart meiner an der grenze zwischen Obersachsen und der Lausitz gelegenen heimat ist braut allgemein volksüblich für die verlobte bis zum hochzeitstage incl: andere verwendungsarten des wortes, insbesondere für ein illegitimes verhältnis, oder für junge frau, sind daselbst absolut unbekannt. Dagegen scheinen die süddeutschen und westdeutschen mundarten den früheren gebrauch festzuhalten. Dér Schweizer Josua Maaler verzeichnet noch nach Luther, wie oben erwähnt, den älteren gebrauch. J. Grimm; DWb. 2, 332 führt an, 'dass in deutschen landstrichen die neuvermählte den namen braut ein jahr lang, oder bis zu den nächsten ostern fortführt ${ }^{2}$ ), oder selbst bis sie kinder. geboren hat: ich "bin braut habe von meinem ehemann noch keine kinder. In H. Fischers Schwäbischem wb. 1, 1372 wird als schwäbisch verzeichnet sie ist braut von ihm (unehelich geschwängert). Im

1) Valentinus = 14. februar, der 1479 auf einen sonntag fiel.

2) Vgl. Hertel, Thüringer sprachschatz s. 73. 
allgemeinen kann man sagen, dass nach den dialektwörterbüchern zu urteilen die mundarten der süd- und westdeutschen gebiete die alten bedeutungen weiterführen: insbesondere ist für das legitime verhältnis braut hauptsächlich in beziehung auf die hochzeit in gebrauch. Nur sehr ausnahmsweise, und vielleicht durch einwirkung der schriftsprache tritt die ausdehnung auf die zeit des verlobtseins hervor. ${ }^{1}$ )

Von dieser abschweifung auf die geschichte der nhd. bedeutungsentwicklung, die freilich nur ein versuch ist und ohne systematische sammlung aus schriftsprache und mundarten nicht zu ende geführt werden kann, kehren wir zurück zu der von uns festgestellten grundbedeutung und sehen $z u$, ob von dieser aus nun die frage nach der etymologischen verknüpfung des germ. brüpi-, brüzi- mit worten der verwanten idg. sprachen sich beantworten lasse. Nachdem die auf grund der verlobungstheorie gemachte combination schon oben s. 36 ausgeschaltet ist, bleiben immer noch eine anzahl von hypothesen zu erörtern. Schon aus lautlichen gründen ist die Bopp'sche von J. Grimm im DWb. 2, 331 vertretene anknüpfung

1) Freilich sind die mundartenwörterbücher hinsichtlich der begriffsbestimmung von braut und bräutigam mangelhaft: es wäre wünschenswert, dass das verhältnis zum schriftsprachlichen bedeutungsumfang überall genau erörtert würde. Hervorzuheben ist, dass in ganz Süddeutschland statt der schriftsprachlichen ausdrücke die bezeichnungen hochzeiterin und hochzeiter weit verbreitet sind. Vgl. z. b. Schmeller-Frommänn 1,371 (braut, 'öfter hört man indessen beim gemeinen volke die hochzeiterinn'); Schöpf, 'Tirol. idiot. 269; Martin-Lienhart, Elsäss. wb. 2, 205, und für das südlichste Oberhessen Vilmar, Kuih. idiot. 172. Mir fiel es in Giessen einmal bei einem dienstmädchen dieses gebiets auf, dass sie stets von ihrem hochzeiter sprach, zur bezeichnung ihres verlobten, also im sinne unseres schriftsprachlichen bräutigam. Es hängt dies vielleicht damit zusammen, dass in diesen gebieten braut, brönttigam, wo sie überhaupt sich gehalten haben, noch auf dem altdeutschen bedeutungscentrum ruhen. - Für die Pfalz bezeugt die angabe vou $\mathrm{Ph}$. Lenz (Vergleich. wörterbuch der nhd. sprache $u$. d. Handschuhsheimer dialekts, Baden-Baden 1898, s. 13): 'braut f. meist hóxtsaitnn, neuerdings auch praut' und 'bräutigam $\mathrm{m}$. meist hóxtsaitio, nenerdings auch práitikam', dass hier braut und bräutigäm nur durch den einfluss der hochsprache um sich greifen, während hochzeiterin und hochzeiter die einheimischen ausdrücke sind. Aehnlich dürfte es auch in den übrigen sïddeutschen mundarten liegen, wenn braut, bräıtigam nicht in der alten, sondern in der nhd. bedeutung angewendet werden. 
an aind. prāudhā 'die heimgefïhrte', sowie die von Bugge, Beitr. 13, 184 aufgestellte deutung als idg. par-ıdhi-s 'die heimgeführte' definitiv abzuweisen. Und zwar nicht nur mit

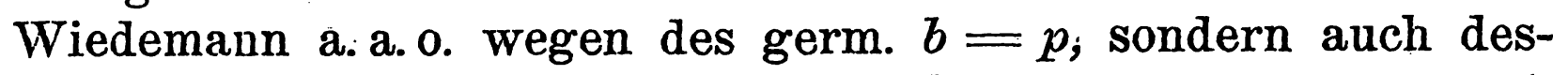
halb, weil der durch das got. brûpi- bezeugte germ. grammat. wechsel nur auf idg. $t$ zurückführen kann.

Im vordergrunde steht jetzt nach Wiedemanns eingehender befürwortung die zusammenstellung von brüpi- mit lit. martì 'braut, junge frau, schwiegertochter', kret. $\mu \alpha$ ó Aber selbst wenn man zugibt, dass der germ. anlaut or hier auf idg. $m r$ zurückgehe, so stimmen die wörter. doch sonst nicht lautlich überein. Uhlenbeck hat Beitr. 30, 272 mit recht geäussert: 'an verwantschaft von $6 r \bar{u} p s$ mit lit: martì ist wegen des germ. $\bar{u}$ nicht zu denken.' Und wenn Wiedemann krimgot. miarzus ${ }^{1}$ ) dazu stellt, so ist dieses ja grade ein gegenbeweis gegen die etymologie, da dadurch klar wird, dass sowol die litauische anlautsform, wie der stammvocal genaue germ. entsprechung in marzus fanden. Hinsichtlich der bedeutung kommt Wiedemann unter abweisung von 'integra, intacta'

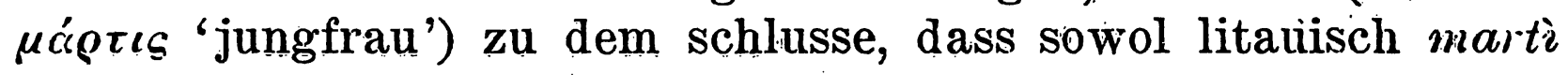
als germ. brūđi- auf die grundlage 'mannbares weib' zurückzuführen sei. Für germ. brüpi müssen wir das jedenfalls zurückweisen, da dessen absolute anwendung, ohne beziehung auf den verkehr mit dem manne, nur hie und da durch poetische metonymie begegnet.

Mir ist es nicht zweifellhaft, dass die zuerst 1838 von L. Döderlein begründete, von Wiedemann s. 205 besprochene zusammenstellung mit lat. Frütis die einzig haltbare combination ist. Frutis ist als ein italischer beiname der. Venus

1) Wenn ïbrigens Wiedemann s. 206 krimgot. marzus mit 'braut' übersetzt, so ist das ein versehen. Bei Pusbeck ist vielmehr marzus. nuptiae überliefert, während darauf folgt schıos sponsa, dessen jetzt beliebte zurückführung auf got. swēs Löwe (Die reste der Germanen s. 175) mit recht bekämpft. Meiner meinung nach hat nur die schon öfter vorgebrachte vermutung etwas für sich, wonach schuos (infolge von schreiboder druckifehler) für schnos stehe. Das würde dann nach den gesetzen der krimgot. lautlehre ( $v$ gl. Löwe s. 136 ff.) ganz correcte entsprechung eines altgot. snu $z \hat{o}$ sein können, das wir oben s. 38 für das got. schon in anspruch genommeu haben. Busbecks sponsa würde dann natürlich nicht im altlateinischen, sondern im mlat. sinne als 'neuvermähltẹ' zu verstehen sein. 
überliefert (bei Solin): Venus mater quae Frıtis dicitur: sie wird daselbst als die göttin erwähnt, welcher Aeneas in agro Laurenti (also in Latium) das aus Sicilien mitgebrachte Aphroditebild weiht. Die zweite nachricht stammt aus Festus bei Paulus diac., wo Frutinal als templum Veneris Fruti genannt wird. Nichts spricht für etruskische herkunft des namens (was man nach Otfr. Müller früher annahm), und die alte vermutung Scaligers, dass er aus Aphrodite umgebildet sei, wird schon durch das lange $\bar{u}$ widerlegt, vgl. Walde, Lat. etymol. wörterbuch, Heidelberg_1906, s. 249. Vielmehr ist Frutis als eine der originalitalischen göttinnen aufzufassen, mit welchen die griechische Aphrodite, ebenso wie mit der römischen Venus, nachträglich identificiert wurde. Vgl. Wissowa, Religion und kultus der Römer (München 1902) s. 236: 'Aphrodite wurde in Rom mit Venus, bei den Oskern mit der dort heimischen Herentas, anderswo mit einer sonst verschollenen göttin Frutis gleichgesetzt.' ') Und dieses Frutis wird von Walde a.a.o. nach dem vorgange Corssens (Aussprache 22, 206) mit lat. frutex 'als bezeichnung der fruchtbarkeit' verknüpft. Dazu stimmt auch die bezeichnung als beiname der 'Venus mater' bei Solin.

Mit Frütis trifft nun buchstäblich das urgotische brü.pis zusammen bis auf die declinationsklasse. Und auch sachlich ist alles in ordnung, wenn das germanische wort seiner grundbedeutung nach als 'die sich begattende, die befruchtete, die fruchtbare' aufgefasst werden darf, wie sich das aus unserer betrachtung ergeben hat. Es wird nun die weitere vermutung gestattet sein, dass altitàlisch frütis ursprünglich ein dem germ. brüpi-, brū $\bar{i}$ - paralleles appellativum war, welches zum götternamen wurde. Die grosse zahl von auffälligen wortübereinstimmungen zwischen italisch und germanisch (vgl. Hirt, Zs. fdph. 29, $289 \mathrm{ff}$.) wäre sonach um ein schlagendes beispiel vermehrt.

1) Auch nach Preller, Röm. mythologie ${ }^{3}$ 1, 43. ff. ist Iìntis eine echt altlateinische göttin (vgl. noch Jordans anm. s. 436,4). Es ist also schon sachlich ungerechtfertigt, die etymologie des wortes anderswo, als im italischen zu suchen.

W. BRAUNE. 